\title{
Comparação das taxas de prenhez entre receptoras com corpos lúteos \\ cavitários ou compactos após protocolo de sincronização com cloprostenol ou transferência de embriões em tempo fixo
}

\author{
Comparison the pregnancy rates between bovine recipients with \\ cavitary or compact corpus luteum after cloprostenol or fixed time \\ embryo transfer
}

\author{
Thales Ricardo Rigo Barreiros ${ }^{1 *}$; Wanessa Blaschi'; Edmilson Antônio Borsato \\ Heitor Ênio Ludwig ${ }^{3}$; Dieggo Ricardo Meira da Silva ${ }^{3}$; Marcelo Marcondes Seneda ${ }^{4}$
}

\section{Resumo}

O objetivo deste experimento foi avaliar as taxas de prenhez em receptoras com corpo lúteo (CL) compacto ou cavitário, submetidas a protocolos com cloprostenol ou transferência em tempo fixo. Foram utilizadas 203 novilhas mestiças. No primeiro grupo (G-PGF, $n=103$ ), os animais com CL previamente detectados por ultra-sonografia transretal receberam $500 \mathrm{mg}$ de Cloprostenol (Sincrocio, Ouro fino, Brasil), por via IM e permaneceram sob observação de cio por 72 horas. No segundo grupo (G-P4, n=100), as novilhas receberam um dispositivo intravaginal de progesterona (CIDR, Pfizer, Brasil) simultaneamente à aplicação IM de $2 \mathrm{mg}$ de benzoato de estradiol (BE); (Estrogin, Farmavet, Brasil). No oitavo dia, os dispositivos foram retirados e os animais receberam $500 \mathrm{mg}$ de Cloprostenol (Sincrocio, Ouro fino, Brasil) por via IM. Após 24 horas, os animais receberam uma aplicação IM de $1 \mathrm{mg}$ de BE. A avaliação ultra-sonográfica do CL foi realizada 10 dias e 17 dias após o início dos tratamentos, respectivamente para os grupos G-PGF e G-P4. Todas as receptoras com CL receberam embriões produzidos in vitro. O diagnóstico de gestação foi realizado 23 dias depois, por ultra-sonografia. Os resultados foram analisados pelo teste do Quiquadrado. Não houve diferença significativa nas taxas de aproveitamento de receptoras entre os grupos G-PGF e G-P4 (72,8\%; 75/103; 79\% ;79/100). Detectou-se CL cavitário em 21,3\% (16/75) das receptoras do G-PGF e 22,7\% (18/79) para o grupo G-P4 (P>0,05). Entre as receptoras com CL cavitário ou compacto, não houve diferença na taxa de prenhez $41,1 \%(14 / 34)$ e 38,3\% (46/120), respectivamente. Os resultados demonstraram que não houve diferença nas taxas de prenhez de receptoras com corpos lúteos cavitários, em comparação às receptoras com CL compactos, independentemente dos tratamentos realizados.

Palavras-chave: Ultra-som, corpo lúteo, transferência de embriões

\footnotetext{
1 Departamento de Veterinária e Produção Animal, UENP/FALM, Bandeirantes-Pr. E-mail: thalesrigo@yahoo.com.br.

2 Departamento de Patologia Geral, UENP/FALM, Bandeirantes-Pr.

${ }^{3}$ Embriogen, Campo Mourão, PR.

${ }^{4}$ Departamento de Clínicas Veterinárias, CCA, UEL.

* Autor para correspondência
} 


\begin{abstract}
The aim of this work was to evaluate pregnancy rates in bovine embryo recipients with compact and cavitary corpus luteum. Two hundred and three crossbred heifers were divided in two groups. In the first group (G-PGF, $\mathrm{n}=103$ ), the animals received $500 \mathrm{mg}$ de Cloprostenol IM (Sincrocio, Ouro fino, Brasil) after CL identification by ultrasonography. After that, heifers were detected for heat the next 72 hours. The animals from second group (G-P4, $\mathrm{n}=100$ ) received an intravaginal progesterone device (CIDR, Pfizer, Brasil) and $2 \mathrm{mg}$ estradiol benzoate (Estrogin, Farmavet, Brasil) IM, in random stages from estrus cycle. On the $8^{\text {th }}$ day the devices were removed and the animals received $500 \mathrm{mg}$ of Cloprostenol IM. After $24 \mathrm{~h}$ all animals received $1 \mathrm{mg} \mathrm{BE}$, IM. The CL was identified and evaluated at 10 and 17 days after the beginning of the treatment in G-PGF and G-P4, respectively. All the heifers with CL received IVP embryos. The pregnancy diagnosis was made by transrectal ultrasonography after 23 days. The results were analyzed by Chi-square. There were no significant difference in number of heifers ready to embryo transfer in G-PGF and G-P4, respectively 72.8\% (75/103) e 79\% (79/100); (P>0.05)Twenty one percent (16/ $75)$ had cavitary CL in G-PGF and 22.7\% (18/79) in G-P4 (P>0.05). Among all embryo recipients with a cavitary or compact CL there was no difference on pregnancy rates $(41.1 \% 14 / 34$ and $38.3 \%$ 46/120, respectively, $\mathrm{P}>0.05$ ), regardless of protocol treatment.
\end{abstract}

Key words: Corpus luteum, ultra-som, embryo transfer

\section{Introdução}

Atualmente, dentre as alternativas propostas para aumentar o aproveitamento dos gametas em fêmeas bovinas e acelerar os programas de melhoramento genético, destacam-se a produção in vivo através da indução de múltipla ovulação e transferência de embriões (MOET) e a produção in vitro de embriões (PIVE); (MERTON et al., 2003). Além do aprimoramento e da associação de técnicas de produção de embriões, o sucesso dos programas de transferência de embriões depende também da disponibilidade de receptoras aptas à inovulação (BOUSQUET et al., 1999; MARQUES; REIS; BARUSELLI, 2004).

O uso de protocolos de sincronização de estro ou de ovulação em receptoras de embrião tem como objetivo a obtenção de animais em fase do ciclo estral compatível com a fase de desenvolvimento embrionário. Os métodos disponíveis para o tratamento de receptoras de embriões incluem a indução de estro com o uso da prostaglandina F $2 \alpha$ ou seus análogos sintéticos e o emprego de protocolos de sincronização da ovulação para transferência de embriões em tempo fixo (TETF); (MARQUES; REIS; BARUSELLI, 2004). A utilização de análogos da prostaglandina para indução de estro é um dos métodos menos dispendiosos para obtenção de receptoras aptas à transferência de embriões. Entretanto, existem algumas condições imprescindíveis para seu uso, tais como, a detecção do corpo lúteo por palpação retal ou ultra-sonografia e observação do comportamento estral nos dias subseqüentes ao tratamento (BO; ADAMS; MAPLETOFT, 2000). Um outro aspecto relevante é a variação na manifestação do estro e ovulação. Kastelic, Knopf e Ginther (1990a) trabalharam com aplicação de $25 \mathrm{mg}$ de dinaprost em novilhas no $5^{\circ}$ ou no $12^{\circ}$ dia após a ovulação e obtiveram a ocorrência das ovulações, respectivamente, 3 e 4, 5 dias após o início do tratamento. Portanto, a aplicação exógena de agentes luteolíticos é eficaz apenas para indução do estro, porém sem controlar o crescimento folicular, fato que pode reduzir o aproveitamento das fêmeas tratadas para transferência de embriões.

Os protocolos com benzoato de estradiol e progesterona intravaginal por um período de 7 a 9 dias permitiram a sincronização do crescimento folicular e o crescimento de uma nova onda folicular 4 a 5 dias após o início do tratamento (BO et al., 1995; BO; ADAMS; MAPLETOFT, 2000). No momento da retirada dos dispositivos a administração de uma dose luteolítica de PGF $2 \alpha$ ou seus análogos e de uma dose de 0,5 a $1 \mathrm{mg}$ de benzoato de estradiol, 24 horas depois, asseguraram a ocorrência de 
ovulação (BURKE; BOLAND; MACMILLAN, 1999; DISKIN; AUSTIN; ROCHE, 2002). Borsato et al. (2004) avaliaram a taxa de sincronização em novilhas cruzadas Bos taurus $x$ Bos indicus tratadas com $1 \mathrm{mg}$ de benzoato de estradiol e dispositivos intravaginais novos ou reutilizados e encontraram $86,8 \%$ (86/99) dos animais com folículos com diâmetro de 8 a $9 \mathrm{~mm}$. As taxas de ovulação descritas com uso destes protocolos estão em torno de 75 a $80 \%$, 64 a 72 horas após a retirada dos dispositivos de progesterona (HANLON et al., 1997; MARTINS et al., 2005). Em razão de taxas adequadas de ovulação, o uso destes protocolos permitiu a transferência de embriões em tempo fixo (TETF) sem prévia observação de cio, empregando apenas a avaliação do corpo lúteo no momento da inovulação dos embriões. Além de facilitar consideravelmente o manejo, as taxas de aproveitamento estão em torno de $70 \%$ dos animais tratados para protocolos de TETF (BARUSELLI et al., 2000; TRIBULO et al., 2000).

Alguns pesquisadores demonstraram que corpos lúteos cavitários são encontrados entre 40 e $80 \%$ dos intervalos interovulatórios examinados para vacas e novilhas tratadas com norgestomet ou prostaglandina e que as concentrações de progesterona não foram influenciadas pela presença da cavidade (KASTELIC; PIERSON; GINTHER, 1990b; SPELL et al., 2001; MARQUES et al., 2002). Entretanto o aproveitamento de receptoras com corpos lúteos cavitários tem gerado controvérsias, particularmente por um suposto aumento na incidência deste tipo de CL em protocolos sem observação de cio. As relações entre o tamanho do folículo pré-ovulatório e posteriormente do corpo lúteo foram estudas em receptoras de embrião por Baruselli et al. (2001). Foi encontrado aumento da concentração de progesterona de acordo com o aumento do tamanho do corpo lúteo obtido após o tratamento com progesterona e estradiol. Segundo Binelli et al. (2001) a obtenção de corpos lúteos maiores em receptoras pode aumentar as taxas de prenhez nos programas de transferência de embriões.

O objetivo deste experimento foi comparar as taxas de prenhez de novilhas com corpo lúteo compacto ou cavitário em novilhas tratadas com cloprostenol ou dispositivos intravaginais de progesterona e benzoato de estradiol, bem como suas respectivas taxas de aproveitamento.

\section{Material e Métodos}

O presente experimento foi conduzido durante os meses de outubro e novembro de 2004. Foram utilizadas como receptoras de embrião, 203 novilhas Bos taurus x Bos indicus ( $1 / 2$ sangue Simental x 1/2 sangue Nelore), cíclicas e com escore da condição corporal entre 3,5 e 4 (LOWMAN; SCOTT; SOMERVILLE, 1976). Estas novilhas eram manejadas extensivamente em pastagem de Cynodon nlemfuensis e recebiam suplementação mineral ad libitum com $9 \%$ de fósforo, na região de Campo Mourão, Estado do Paraná. Estes animais foram previamente examinados por palpação retal, de modo que todos apresentaram o aparelho reprodutivo morfologicamente normal.

Estas receptoras foram separadas em dois grupos equiparados em relação ao escore da condição corporal. No primeiro grupo (G-PGF, $n=103$ ), os animais com CL detectados por ultra-sonografia transretal (Aloka SSD 500, $5 \mathrm{MHz}$ ) receberam 500 $\mathrm{mg}$ de Cloprostenol sódico (Sincrocio, Ouro fino, Brasil), por via intramuscular (IM) e permaneceram em observação de cio nas próximas 72 horas. No segundo grupo (G-P4, $\mathrm{n}=100$ ), as novilhas receberam, em momento aleatório do ciclo estral, um dispositivo intravaginal de 1,9 $\mathrm{g}$ de progesterona (CIDR, Pfizer, Brasil) simultaneamente à aplicação IM de $2 \mathrm{mg}$ de benzoato de estradiol (Estrogin, Farmavet, Brasil). No oitavo dia, os dispositivos foram retirados e os animais receberam $500 \mathrm{mg}$ de Cloprostenol (Sincrocio, Ouro fino, Brasil) por via IM. Vinte e quatro horas depois, todos os animais receberam uma aplicação de $1 \mathrm{mg}$ de benzoato de estradiol, por via IM. A identificação ultra-sonográfica do corpo lúteo (CL) foi realizada dez dias e dezessete dias após o início dos tratamentos, respectivamente para os grupos G-PGF e G-P4. Os tratamentos utilizados estão representados na Figura 1. 

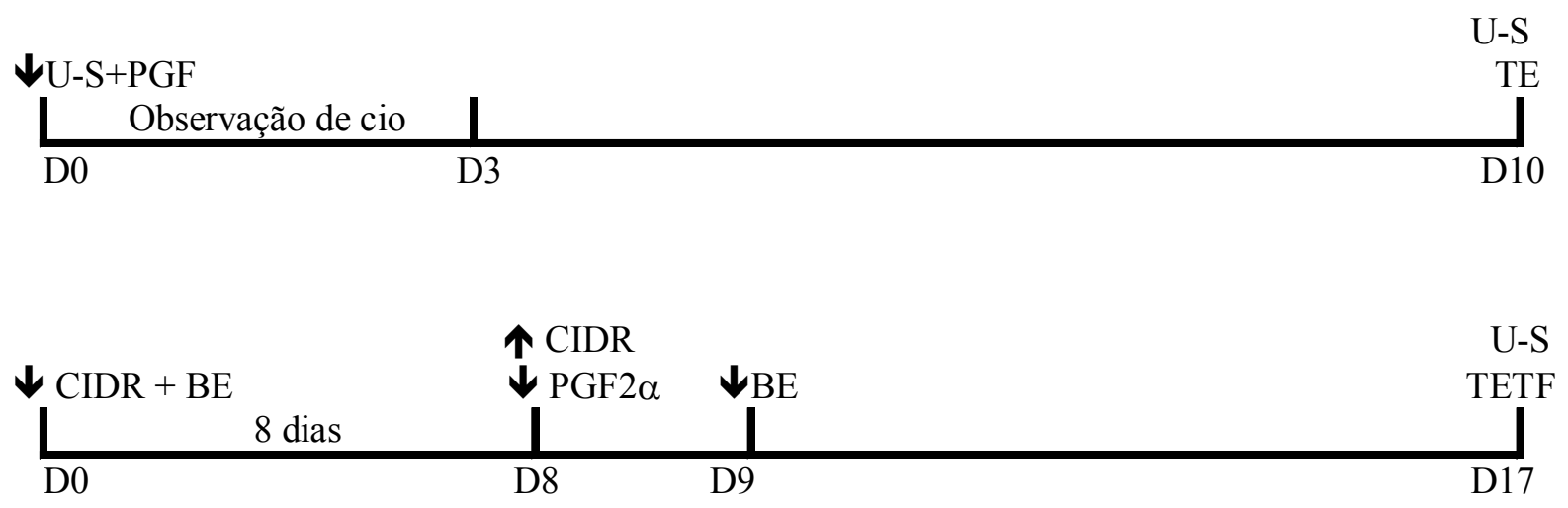

Figura 1. Representação esquemática dos protocolos de sincronização de cio e transferência de embriões em tempo fixo utilizados em receptoras Bos taurus x Bos indicus.

$\mathrm{Na}$ avaliação das receptoras foram verificadas a presença do corpo lúteo e as características ultrasonográficas, sendo classificados em cavitários e compactos, respectivamente para a presença ou não de cavidade. Independente das características ultrasonográficas encontradas, todas as receptoras com CL foram inovuladas pelo método transcervical com embriões Bos taurus indicus produzidos in vitro. $\mathrm{O}$ diagnóstico de gestação foi realizado aos 30 dias de gestação por ultra-sonografia transretal.

A análise estatística foi realizada aplicando-se o teste do Qui-quadrado corrigido de Yates $\left(\chi^{2}\right)$, com nível de significância igual a $5 \%(\mathrm{P}<0,05)$, utilizando-se o software EPI INFO versão 6.04 (DEAN et al., 1997).

\section{Resultados e Discussão}

A taxa de aproveitamento foi estabelecida pelo número de receptoras consideradas aptas em relação a todas as tratadas em cada grupo e a taxa de prenhez foi obtida a partir da relação entre as novilhas gestantes e as novilhas aptas à inovulação. Na avaliação ultra-sonográfica para detecção do corpo lúteo, não houve diferença significativa nas taxas de aproveitamento de receptoras entre os grupos G-PGF e G-P4, resultando, respectivamente, em 72,8\% (75/ 103) e 79\% (79/100); (P>0,05); (Tabela 1).

Tabela 1. Taxas de aproveitamento obtidas em receptoras sincronizadas com cloprostenol (G-PGF) ou CIDR (G-P4) e benzoato de estradiol.

\begin{tabular}{ccc} 
Grupo & $\mathrm{N}$ & Taxa de aproveitamento $(\%)$ \\
\hline $\mathrm{G}-\mathrm{PGF}$ & 103 & $72,8(75 / 103)$ \\
$\mathrm{G}-\mathrm{P} 4$ & 100 & $79(79 / 100)$ \\
\hline
\end{tabular}

$(\mathrm{P}>0,05)$

As taxas de aproveitamento obtidas no presente experimento foram semelhantes às encontradas por Tribulo et al. (2000) que não observaram diferença significativa $(\mathrm{P}>0,05)$ nas taxas de aproveitamento em vacas submetidas a duas aplicações IM de PGF com intervalo de 14 dias $(60 \% ; 60 / 100)$ ou naquelas que receberam tratamento com CIDR e benzoato de estradiol $(59 \%$; 59/100) para transferência de embriões em tempo fixo. Contrariamente, Baruselli et al. (2000) encontraram acréscimo de $27 \%$ $(\mathrm{P}<0,05)$, no aproveitamento de receptoras tratadas com o protocolo Ovsynch em relação às do grupo tratado com cloprostenol. 
Os corpos lúteos foram classificados em compactos e cavitários, desta forma detectou-se CL cavitário em $21,3 \%(16 / 75)$ das receptoras do G-PGF e $22,7 \%(18 / 79)$ para o grupo G-P4 $(\mathrm{P}>0,05)$ como demonstrado na Figura 2.

O percentual de corpos lúteos cavitários encontrado no presente experimento foi inferior ao encontrado por outros pesquisadores. Marques et al. (2002) observaram 43,7\% (14/32) em novilhas cruzadas Bos taurus x Bos indicus tratadas com duas doses de $150 \mathrm{mg}$ de cloprostenol com intervalo de 13 dias. Kastelic, Pierson e Ginther (1990b) avaliaram diariamente por ultra-sonografia, 80 intervalos interovulatórios em novilhas Holandesas e encontraram 79\% (63/80) de corpos lúteos cavitários. O mesmo percentual foi encontrado por Spell et al. (2001) em novilhas tratadas com dinaprost ou dispositivos auriculares de norgestomet.

As receptoras que apresentavam CL compacto e cavitário foram agrupadas e compararam-se as taxas de prenhez obtidas, como está representado na Tabela 2.

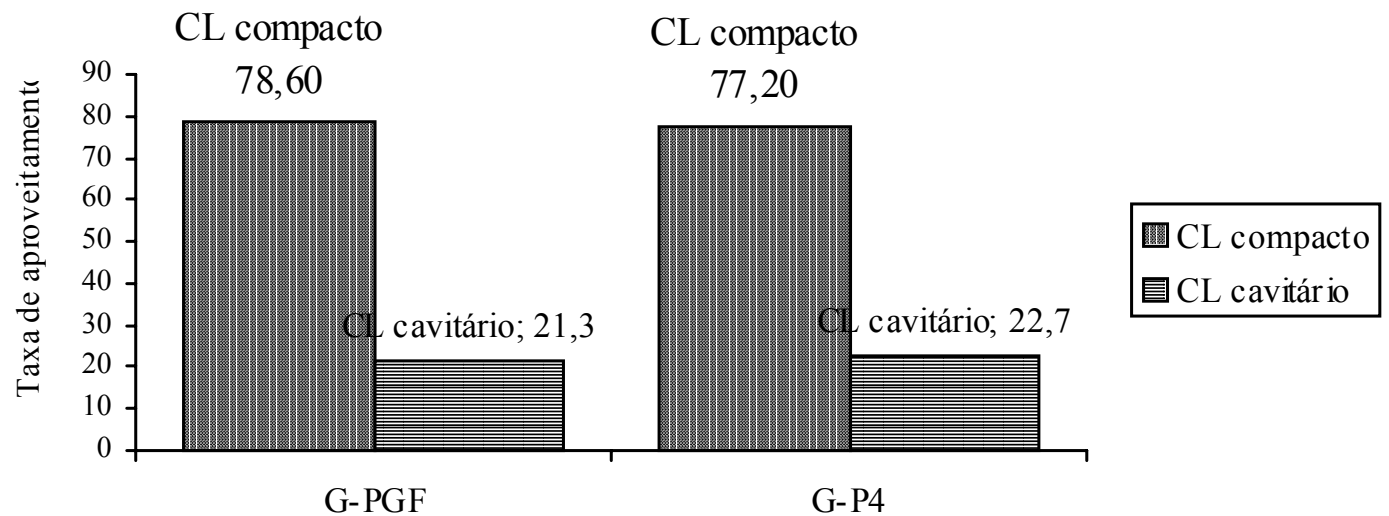

Figura 2. Taxas de aproveitamento (\%), segundo o tipo de CL avaliado por ultra-sonografia em novilhas Bos taurus $\mathrm{x}$ Bos indicus tratadas com cloprostenol ou CIDR e benzoato de estradiol.

Tabela 2. Taxas de prenhez, segundo o tipo de corpo lúteo (CL) em receptoras sincronizadas com cloprostenol ou CIDR e benzoato de estradiol e inovuladas com embriões produzidos in vitro.

\begin{tabular}{ccc}
\hline Tipo de CL & $\mathrm{N}$ & Taxa de prenhez $(\%)$ \\
\hline Compacto & 120 & $38,3(46 / 120)$ \\
Cavitário & 34 & $41,1(14 / 34)$ \\
\hline
\end{tabular}

$(\mathrm{P}>0,05)$

Entre as receptoras com CL cavitário ou compacto, não houve diferença na taxa de prenhez $41,1 \%(14 / 34)$ e $38,3 \%(46 / 120)$, respectivamente, $(\mathrm{P}>0,05)$. Das 34 novilhas gestantes e que possuíam CL cavitário, não foi observada diferença nas taxas de prenhez entre G-PGF e G-P4. Estes resultados estão de acordo com os obtidos por Spell et al. (2001) que não encontraram diferença nas taxas de prenhez de receptoras que receberam embriões produzidos in vivo transferidos a fresco ou previamente congelados com glicerol. Um outro aspecto observado por Kastelic, Pierson e Ginther (1990b), foi a perda das cavidades dos corpos lúteos em novilhas gestantes fato que não indica importância funcional deste achado ultra-sonográfico. Adicionalmente, em outros estudos não houve diferença significativa na concentração de progesterona em receptoras com corpos lúteos cavitários ou compactos no momento da inovulação (SPELL et al., 2001; MARQUES et al., 2002). 
A partir destes resultados, destaca-se a semelhança nas taxas de prenhez de receptoras com corpos lúteos cavitários, em comparação às receptoras com CL compactos, independentemente da receptora ter sido submetida a protocolos convencionais com o uso de agentes luteolíticos ou de transferência de embriões em tempo fixo. Adicionalmente, não foram encontradas diferenças nas taxas de aproveitamento de receptoras, após tratamentos com cloprostenol ou progesterona intravaginal.

\section{Referências}

BARUSELLI, P. S.; MARQUES, M. O.; CARVALHO, N. A. T.; VALENTIM, R.; CARVALHO FILHO, A. F.; MADUREIRA, E. H.; COSTA NETO, W. P. Aumento da taxa de prenhez em receptoras de embrião bovino pela utilização do protocolo "Ovsynch" com inovulação em tempo fixo. In: REUNIÃO ANUAL DA SOCIEDADE BRASILEIRA DE TRANSFERÊNCIA DE EMBRIÕES, 15. 2000, Rio Quente. Resumos... Porto Alegre: Universidade Federal do Rio Grande do Sul, 2000. p.216.

BARUSELLI, P. S.; MARQUES, M. O.; HOFFMANN, E. M.; COSTA NETO, W. P.; GRANDINETTI, R. R.; BO, G. A. Incresead pregnancy rates in embryo recipients treated with CIDR-B devices. Theriogenology, Stoneham, v.55, p.355, 2001 .

BINELLI, M.; THATCHER, W. W.; MATTOS, R.; BARUSELLI, P. S. Antiluteolytic strategies to improve fertility in cattle. Theriogenology, Stoneham, v.56, n.9, p.1451-1463, 2001.

BO, G. A.; ADAMS, G. P.; MAPLETOFT, R. J. Dinámica folicular ovárica en el bovino. In: SIMPÓSIO SOBRE CONTROLEFARMACOLÓGICODOCICLOESTRALEM RUMINANTES, 1., 2000, São Paulo. Anais... São Paulo: USP, 2000. p.12-34.

BO, G. A.; ADAMS,G. P.; CACCIA, M.; MARTINEZ, M. F.; PIERSON, R. A.; MAPLETOFT, R. J. Ovarian follicular wave emergence after treatament with progesterone and estradiol in cattle. Animal Reproduction Science, Amsterdam, v.39, n.3, p.193-204, aug. 1995.

BORSATO, E. A.; LUDWIG JÚNIOR, H. E.; RUBIN, K. C. P.; SAUT, J. P.; BARREIROS, T. R. R.; SENEDA, M. M. Relação entre o tamanho do folículo ovulatório e taxa de concepção em novilhas Bos taurus X Bos indicus submetidas a inseminação artificial em tempo fixo. Revista Brasileira de Reprodução Animal, Belo Horizonte, v.28, p.137-142, 2004.
BOUSQUET, D.; TWAGIRAMUNGU, H.; MORIN, N.; BRISSON, C.; CARBONEAU, G.; DUROCHER, J. In vitro embryo production in the cow: an effective alternative to the conventional embryo production approach. Theriogenology, Stoneham, v.51, n.1, p.59-70, jan. 1999.

BURKE, C. R.; BOLAND, M. P.; MACMILLAN, K. L. S. O. Ovarian responses to progesterone and oestradiol benzoate administered intravaginally during dioestrus in cattle. Animal Reproduction Science, Amsterdam, v.55, p.23-33, 1999.

DEAN, A, G.; DEAN, J. A.; COULOMBIER, D.; BURTON, A. H.; BRENDEL, K. A.; SMITH, D. C.; DICKER, R. C; SULLIVAN, K. M.; FAGAN, R.F.; ARNE, T.G. Epi info versão 6.094b. Disponível em <www.cdc.gov/epinfo./ 6i6faq.htm> Acesso em: 28 set. 2006.

DISKIN, M. G.; AUSTIN, E. J.; ROCHE, J. F. Exogenous hormonal manipulation of ovarian activity in cattle. Domestic Animal Endocrinology, Stoneham, v.23, n1/2, p.211-228, 2002.

HANLON, D. W.; WILLIAMSON, N. B.; WICHTEL, J. J.; STEFFERT, I. J.; CRAIGIE,A. L.; PFEIFFER, D. U. The effect of estradiol benzoate administration on estrous response and synchronized pregnancy rate in dairy heifers after treatment with exogenous progesterone. Theriogenology, Stoneham, v.45, n.4, p.775-785, mar. 1997.

KASTELIC, J. P.; KNOPF, L.; GINTHER, O. J. Effect of day of prostaglandin f2a treatment on selection and development of the ovulatory follicle. Animal Reproduction Science, Amsterdam, v.23, n.3, p.169-180, nov. 1990a.

KASTELIC, J. P.; PIERSON, R. A.; GINTHER, O. J. Ultrasonic morphologic of corporea lutea and central cavities during the estrous cycle and early pregnancy in heifers. Theriogenology, Stoneham, v.34, n.3, p.487-498, sep. 1990b.

LOWMAN, B. G.; SCOTT, N. A.; SOMERVILLE, S. H. Condition scoring of catlle. Edimburg: East of Scotland College of Agriculture. Animal production. Advisory and Development Department, 1976. (Bulletin, 6). p.1-31.

MARQUES, M. O.; ARRUDA, R. P.; MADUREIRA, E. H.; OLIVEIRA, C. A.; BARUSELLI, P. S. Efeito da cavidade do corpo lúteo na concentração plasmática de progesterona em novilhas Bos taurus x Bos indicus receptoras de embrião. Revista Brasileira de Reprodução Animal, Belo Horizonte, v.26, p.238-240, 2002.

MARQUES, M. O.; REIS, E. L.; BARUSELLI, P. S. Sincronização de receptoras para inovulação em tempo fixo. In: SIMPÓSIO INTERNACIONAL DE REPRODUÇÃO ANIMAL APLICADA, 1., 2004, Londrina. Anais... Londrina: USP, 2004. p.199-211. 
MARTINS, C. M.; CASTRICINI, E. S. C.; SÁ FILHO, M. F.; GIMENES, L. U.; BARUSELLI, P. S. Dinâmica folicular de vacas Nelore tratadas com cipionato ou benzoato de estradiol em protocolo de inseminação artificial em tempo fixo. Acta Scientiae Veterinariae, Porto Alegre, v.33, supl.1, p.285, 2005.

MERTON, J. S.; ROOS, A. P. W.; MULLAART, E.; RUIGH, L.; KAAL, L; VOS, P. L. A. M.; DIELEMAN, S. J. Factors affecting oocyte quality and quantity in commercial application of embryo technologies in the cattle breeding industry. Theriogenology, Stoneham, v.59, n.2, p.651-674, jan. 2003.
SPELL, A. R.; BEAL, W. E.; CORAH, L. R.; LAMB, G. C. Evaluating recipient and embyo factors that affect pregnancy rates of embryo transfer in beef cattle. Theriogenology, Stoneham, v.56, n.2, p.287-297, jul. 2001.

TRIBULO, H.; BO, G. A.; GATTI, G.; TEGLI, J. C.; CUTAIA, L.; MORENO, D.; BRITO, M.; TRIBULO, R. Pregnancy rates in embryo recipients treated with estradiol benzoate and CIDR-B vaginal devices to eliminate the need for estrus detection. In: INTERNATIONAL CONGRESS ON ANIMAL REPRODUCTION, 14., 2000, Stockholm. Anais...Stockholm: ICAR, 2000. v.2, p.115. 